\title{
On Coupled $p$-Laplacian Fractional Differential Equations with Nonlinear Boundary Conditions
}

\author{
Aziz Khan, ${ }^{1}$ Yongjin Li, ${ }^{2}$ Kamal Shah, ${ }^{3}$ and Tahir Saeed Khan ${ }^{1}$ \\ ${ }^{1}$ Department of Mathematics, University of Peshawar, P.O. Box 25000, Khybar Pakhtunkhwa, Pakistan \\ ${ }^{2}$ Department of Mathematics, Sun Yat-Sen University, Guangzhou, China \\ ${ }^{3}$ Department of Mathematics, University of Malakand, Chakdara, Dir (L), Khyber Pakhtunkhwa, Pakistan
}

Correspondence should be addressed to Yongjin Li; stslyj@mail.sysu.edu.cn

Received 2 May 2017; Accepted 17 July 2017; Published 17 August 2017

Academic Editor: Fathalla A. Rihan

Copyright (C) 2017 Aziz Khan et al. This is an open access article distributed under the Creative Commons Attribution License, which permits unrestricted use, distribution, and reproduction in any medium, provided the original work is properly cited.

\begin{abstract}
This paper is related to the existence and uniqueness of solutions to a coupled system of fractional differential equations (FDEs) with nonlinear $p$-Laplacian operator by using fractional integral boundary conditions with nonlinear term and also to checking the Hyers-Ulam stability for the proposed problem. The functions involved in the proposed coupled system are continuous and satisfy certain growth conditions. By using topological degree theory some conditions are established which ensure the existence and uniqueness of solution to the proposed problem. Further, certain conditions are developed corresponding to Hyers-Ulam type stability for the positive solution of the considered coupled system of FDEs. Also, from applications point of view, we give an example.
\end{abstract}

\section{Introduction}

Due to high profile accuracy and usability, FDEs become an area of interest for various fields of scientists and mathematicians. In last few years, some physical phenomenons were described through FDEs and compared with integer order differential equations which have better results, that is why researchers of different areas have paid great attention to study FDEs. The applications of FDEs can be studied in several disciplines including aerodynamics, engineering, electrical circuits, plasma physics, chemical reaction design, turbulent filtration in porous media, and signal and image processing; for further details we refer to [1-5].

Nonlinear operators have vital roles in differential equations; one of the most important operators used in FDEs is the classical nonlinear $p$-Laplacian operator, which is defined as

$$
\begin{aligned}
& \frac{1}{p}+\frac{1}{q}=1, \\
& \phi_{p}(s)=|s|^{p-2} s, \quad p \geq 1, \\
& \phi_{q}(\tau)=\phi_{p}^{-1} .
\end{aligned}
$$

For further details and applications of the nonlinear $p$ Laplacian operators, reader should study [6].

Researchers study different aspects of FDEs involving $p$ Laplacian operators like the existence theory, which has been extensively investigated by using classical fixed point theory. The mentioned theory has been investigated very well for the aforesaid equations of ordinary and partial fractional differential equations. Since $p$-Laplacian operators have been greatly applied in the mathematical modeling of large numbers of real world phenomenons devoted to physics, mechanics, dynamical systems, electrodynamics, and so forth, therefore researchers paid much attention to study such type of differential equation dealing with $p$-Laplacian operators from different aspects including existence theory, multiplicity results, and stability analysis. For instance, Lu et al. [7] discussed Sturm-Liouville boundary value problems (BVP) of FDEs with $p$-Laplacian operator for existence of two or three positive solutions by using fixed point theory. By applying Leggett-William fixed point theorem, the mentioned author studied the following problem:

$$
\begin{aligned}
\mathrm{D}^{\beta}\left(\phi_{p}\left((D)^{\alpha} \mu(x)\right)\right)+\mathfrak{F}(x, \mu(x)) & =0, \quad 0<x<1, \\
j \mu(0)-\eta \mu^{\prime}(0) & =0,
\end{aligned}
$$




$$
\begin{aligned}
\gamma \mu(1)+\delta \mu^{\prime}(1) & =0, \\
D^{\alpha} \mu(0) & =0
\end{aligned}
$$

and also he provided proper example, where $\mathrm{D}^{\alpha}$ and $\mathrm{D}^{\beta}$ denote standard Caputo fractional derivatives with $1<\alpha \leq 2$, $0<\beta \leq 1,1 / p+1 / q=1, \phi_{p}(\tau)=|s|^{p-2} s, p>1, \phi_{q}(\tau)=\phi_{p}^{-1}$, $\rho=\jmath \gamma+\jmath \delta+\eta \gamma>0, \jmath, \eta, \delta, \gamma \geq 0$, and $\mathfrak{F}$ is continuous.

Hu and Zhang [8] have investigated a nonlinear FDE with $p$-Laplacian operator for existence of solution as given by

$$
\begin{aligned}
& \mathrm{D}^{\beta}\left(\phi_{p}\left((D)^{\alpha} \mu(x)\right)\right)+\mathfrak{F}\left(x, \mu(x), \mathrm{D}^{\alpha} \mu(x)\right)=0, \\
& x \in(0,1), \\
& \mathrm{D}^{\alpha} \mu(0)=0=\mathrm{D}^{\alpha} \mu(1),
\end{aligned}
$$

where $0<\alpha, \beta<1,1<\alpha+\beta<2, \mathrm{D}^{\alpha}$ and $\mathrm{D}^{\beta}$ represent standard Caputo fractional derivatives, $\mathfrak{F}$ is continuous and $1 / p+1 / q=1, \phi_{p}(\tau)=|s|^{p-2} s, p>1$, and $\phi_{q}(\tau)=$ $\phi_{p}^{-1}$. Zhi et al. [9] have investigated the existence of positive solutions for the nonlocal BVP of FDEs with $p$-Laplacian operator and illustrated the problem with expressive example. The corresponding problem is given by

$$
\begin{aligned}
\left(\phi_{p}\left(\mathrm{D}^{\alpha} \mu(x)\right)\right)^{\prime \prime} & =\mathfrak{F}\left(x, \mu(x), \mathrm{D}^{\beta} \mu(x)\right), \\
x \in(0,1), & \\
\left.\mu(x)\right|_{x=0} & =\left.\mu^{\prime \prime}(x)\right|_{x=0}=0, \\
\mu(1) & =\int_{0}^{1} g(\tau) \mu(\tau) d \tau, \\
\left(\phi_{p}\left(\mathrm{D}^{\alpha}\right) \mu(0)\right)^{\prime} & =\xi_{1}\left(\phi_{p}\left(\mathrm{D}^{\alpha}\right) \mu\left(\jmath_{1}\right)\right)^{\prime}, \\
\left(\phi_{p}\left(\mathrm{D}^{\alpha}\right) \mu(1)\right) & =\xi_{2}\left(\phi_{p}\left(\mathrm{D}^{\alpha}\right) \mu\left(j_{2}\right)\right),
\end{aligned}
$$

where $\phi_{p}$ is $p$-Laplacian operator and $2<\alpha \leq 3,1<$ $\beta<\alpha-1<2,0<\jmath_{1} \leq \jmath_{2}<1,0 \leq \xi_{1}, \xi_{2}<1$, and $\mathrm{D}^{\alpha}$ expresses Caputo derivative of order $\alpha$. For further study about the existence theory and multiplicity results of $p$-Laplacian operator involved in differential equations, see [10-14].

Using classical fixed point theory needs strong conditions to establish conditions for existence and uniqueness of solutions to FDEs and therefore restrict the applicability to certain classes of FDEs and their systems. To relax the criteria degree theory plays excellent roles for the existence of solutions to FDEs and their systems. Various degree theories including Brouwer and Leray-Schauder were established to deal with the existence theory of differential equations. An important degree theory known as topological degree theory which was introduced by Stamova [15] and later on extended by Isaia [16] has been used to establish existence theory for solutions to nonlinear differential and integral equations. The mentioned method is called prior-estimate method which needs no compactness of the operator and relaxes much the condition for existence and uniqueness of solutions to differential and integral equations. Recently, the aforesaid degree theory has been applied to investigate certain classes of FDEs with boundary conditions; see [17-19].

In recent years another aspect of FDEs which has greatly attracted the attentions of researchers is devoted to the stability analysis of the mentioned equations. Stability analysis plays significant roles in the optimization and numerical analysis of the aforesaid equations. Different kinds of stability have been studied for fractional differential equations including exponential, Mittag-Leffler, and Lyapunov stabilities; see $[15,20,21]$. An important stability was pointed out by Ulam [22], in 1940, which was formally introduced by Hyers [23], in 1941. The aforesaid stability has now been considered in many papers for classical differential equations; see [24-26]. For instance, Urs [27] has investigated the Hyers-Ulam stability for the following coupled periodic BVPs given as

$$
\begin{aligned}
\mu^{\prime \prime}(x)-\mathfrak{F}_{1}(x, \mu(x)) & =\mathfrak{F}_{2}(x, v(x)), \quad x \in[0, T], \\
v^{\prime \prime}(x)-\mathfrak{F}_{1}(x, v(x)) & =\mathfrak{F}_{2}(x, \mu(x)), \\
\left.\mu(x)\right|_{x=0} & =\left.\mu(x)\right|_{x=T}, \\
\left.v(x)\right|_{x=0} & =\left.v(x)\right|_{x=T} .
\end{aligned}
$$

The Hyers-Ulam stability has been investigated for certain FDEs with boundary and initial conditions; see [28-30]. In many situations, Lyapunov type stability and its investigation are very difficult and time-consuming for certain nonlinear fractional differential equations. This is due to the predefined Lyapunov function which is often very difficult to construct for FDEs. Therefore, Hyers-Ulam type stability plays important roles in such a situation. Inspired from the above-mentioned work, in this paper, we study a coupled system of FDEs with nonlinear $p$-Laplacian operator by using topological degree theory. Further, we also investigated some conditions for the Hyers-Ulam stability of the solution to the proposed problem. The proposed problem is given by

$$
\begin{aligned}
& \mathrm{D}^{\beta_{1}} \phi_{p}\left(\mathrm{D}^{\alpha_{1}} \mu(x)\right)+\mathfrak{F}_{1}(x, v(x))=0, \quad x \in(0,1), \\
& \mathrm{D}^{\beta_{2}} \phi_{p}\left(\mathrm{D}^{\alpha_{2}} v(x)\right)+\mathfrak{F}_{2}(x, \mu(x))=0, \quad x \in(0,1), \\
& \left.\mathrm{D}^{\alpha_{1}} \mu(x)\right|_{x=0}=0=\left.\mu(x)\right|_{x=0}=\left.\mu^{\prime \prime}(x)\right|_{x=0}, \\
& \left.\mu^{\prime}(x)\right|_{x=1}=\eta_{1} I_{P}^{\gamma_{1}} \psi_{1}(\mu) \\
& \quad=\frac{\eta_{1}}{\Gamma\left(\gamma_{1}\right)} \int_{0}^{P}(P-\tau)^{\gamma_{1}-1} \psi_{1}(\mu(\tau)) d \tau, \\
& \left.\mathrm{D}^{\alpha_{2}} v(x)\right|_{x=0}=\left.v(x)\right|_{x=0}=\left.v^{\prime \prime}(x)\right|_{x=0}=0, \\
& \left.v^{\prime}(x)\right|_{x=1}=\eta_{2} I_{P}^{\gamma_{2}} \psi_{2}(v) \\
& =\frac{\eta_{2}}{\Gamma\left(\gamma_{2}\right)} \int_{0}^{P}(P-\tau)^{\gamma_{2}-1} \psi_{2}(v(\tau)) d \tau,
\end{aligned}
$$

where $2<\alpha_{i}<3,0<\beta_{i}<1, P, \eta_{i}, \gamma_{i}>0, \psi_{1}, \psi_{2} \in L[0,1]$, and $\mathrm{D}^{\alpha_{i}}$ and $\mathrm{D}^{\beta_{i}}$ where $i=1,2$ stand for Caputo fractional 
derivative, $\phi_{p}(\kappa)=|\kappa|^{p-2} \kappa$ is $p$-Laplacian operator, where $1 / p+1 / q=1, \phi_{q}$ denotes inverse of $p$-Laplacian, and $\mathfrak{F}_{i}$ : $[0,1] \times[0, \infty) \rightarrow(0, \infty), i=1,2$, are continuous functions. Here, we remark that applying degree method to deal with existence and uniqueness and to find conditions for HyersUlam stability to a coupled system of FDEs with $p$-Laplacian operator has not been investigated properly to the best of our knowledge. Therefore thanks to the coincidence degree theory and nonlinear functional analysis greatly developed by Deimling [31], we establish necessary and sufficient conditions for existence and uniqueness as well as for HyersUlam stability corresponding to the aforementioned problem considered by us. We also demonstrate our result through expressive example.

\section{Axillary Results}

Here we recall some special definitions, theorems, and HyersUlam stability results from the literature [1-4] which have important applications throughout this paper.

Definition 1. The integral with fractional order $\alpha>0$ of Riemann-Liouville type is defined for the function $\mathfrak{\mho}$ as

$$
I_{0}^{\alpha} \mathfrak{F}(x)=\frac{1}{\Gamma(\alpha)} \int_{0}^{x}(x-\tau)^{\alpha-1} \mathfrak{F}(\tau) d \tau,
$$

provided that the integral on the right converges pointwise on $(0, \infty)$.

Definition 2. The derivative with fractional order $\alpha>0$ of Caputo type is defined for the function $\mathfrak{F}$ as

$$
D_{0}^{\alpha} \mathfrak{F}(x)=\frac{1}{\Gamma(m-\alpha)} \int_{0}^{x}(x-\tau)^{m-\alpha-1} \mathfrak{F}^{(m)}(\tau) d \tau,
$$

where $m=[\alpha]+1,[\alpha]$ is the integer part of $\alpha$ such that the integral on the right converges pointwise on $(0, \infty)$.

Lemma 3. Let $\alpha>0$ and $\mu \in C(0,1) \cap L^{1}(0,1)$, and then the general solution of FDE

$$
D_{0}^{\alpha} \mu(x)=y(x)
$$

is given by

$$
\begin{aligned}
\mu(x)= & I_{0}^{\alpha} y(x)+A_{0}+A_{1} x+A_{2} x^{2}+\cdots \\
& +A_{m-1} x^{m-1},
\end{aligned}
$$

for some $A_{i} \in \mathbb{R}, i=0,1,2, \ldots, m-1$ and $m$ is smallest integer such that $m \geq \alpha$.

Let $\mathscr{L}$ be the space of all continuous functions $\mu$ : $[0,1] \rightarrow \mathbb{R}$ endowed with a norm $\sup _{x \in[0,1]}\{|\mu(x)|: \mu \epsilon$ $C[0,1]\}$ which is obviously a Banach space. Then the product space denoted by $\mathscr{L}=\mathscr{L}_{1} \times \mathscr{L}_{2}$ under the norms $\|(\mu, v)\|=$ $\|\mu\|+\|v\|$ is also a Banach space which will be used throughout this paper. For the coincidence degree theory and nonlinear functional analysis, we recall the following definitions which can be traced in $[15,16,31]$ as follows.
Definition 4. Let the class of all bounded set of $P(\mathscr{L})$ be denoted by $\aleph$. Then the mapping $\jmath: \aleph \rightarrow(0, \infty)$ for Kuratowski measure of noncompactness is defined as

$$
\begin{aligned}
& J(\hbar)=\inf \{d \\
&>0: \hbar \text { is the finite cover for sets of diameter } \\
&\leq d\},
\end{aligned}
$$

where $\hbar \in \mathrm{N}$.

Proposition 5. The following are the characteristics of Kuratowski measure j:

(1) For relative compact $\hbar$, then Kuratowski measure $\jmath(\hbar)=0$.

(2) For seminorm $\jmath, \jmath(\kappa \hbar)=|\kappa| \jmath(\hbar), \kappa \in \mathbb{R}$, and $\jmath\left(\hbar_{1}+\right.$ $\left.\hbar_{2}\right) \leq \jmath\left(\hbar_{1}\right)+\jmath\left(\hbar_{2}\right)$.

(3) $\hbar_{1} \subset \hbar_{2}$ yields $\jmath\left(\hbar_{1}\right) ; \jmath\left(\hbar_{1} \cup \hbar_{2}\right)=\sup \left\{\jmath\left(\hbar_{1}\right), \jmath\left(\hbar_{2}\right)\right\}$.

(4) $\jmath(\operatorname{conv} \hbar)=\jmath(\hbar)$.

(5) $\jmath(\bar{\hbar})=\jmath(\hbar)$.

Definition 6. Assume that $\varphi: \vartheta \rightarrow \mathscr{U}$ is bounded and continuous mapping such that $\vartheta \subset \mathcal{U}$. Then $\varphi$ is a $\jmath$-Lipschitz, where $\zeta \geq 0$ such that

$$
\jmath(\varphi(\hbar)) \leq \zeta_{\jmath}(\hbar) \text { for all bounded } \hbar \subset \vartheta .
$$

Then $\varphi$ is called strict $\jmath$-contraction under the condition $\zeta<$ 1.

Definition 7 . The function $\varphi$ is $\mathrm{j}$-condensing if

$$
\jmath(\varphi(\hbar))<\jmath(\hbar),
$$

$$
\text { for all bounded } \hbar \subset \vartheta \text { such that } \jmath(\hbar)>0 \text {. }
$$

Therefore $\jmath(\varphi(\hbar)) \geq \jmath(\hbar)$ yields $\jmath(\hbar)=0$.

Further we have $\varphi: \vartheta \rightarrow \mathscr{U}$ which is Lipschitz for $\zeta>0$, such that

$$
\|\varphi(v)-\varphi(\bar{v})\| \leq \zeta\|v-\bar{v}\|, \quad \text { for all } v, \bar{v} \in \mathcal{\vartheta} .
$$

The condition $\zeta<1$ yields that $\varphi$ is a strict contraction.

Proposition 8. The mapping $\varphi$ is $\mathrm{j}$-Lipschitz with constant $\zeta=$ 0 if and only if $\varphi: \mathcal{\vartheta} \rightarrow \mathcal{U}$, which is said to be compact.

Proposition 9. The operator $\varphi$ is j-Lipschitz for some constant $\zeta$ if and only if $\varphi: \vartheta \rightarrow \mathcal{U}$, which is Lipschitz with constant $\zeta$.

Theorem 10. Let $\varphi: \mathscr{L} \rightarrow \mathscr{L}$ be a j-contraction and

$$
\begin{aligned}
\mathscr{G} & =\{z \in \mathscr{L}: \text { there exist } 0 \leq \lambda \leq 1 \text { such that } z \\
& =\lambda \varphi(z)\} .
\end{aligned}
$$

Under the conditions $\mathscr{G} \subset \mathscr{L}$ is bounded for $r>0$ and $\mathscr{G} \subset$ $\hbar_{r}(0)$, with degree

$$
\operatorname{deg}\left(I-\lambda \varphi, \hbar_{r}(0), 0\right)=1, \quad \text { for every } \lambda \in[0,1] .
$$

Then, $\varphi$ has at least one fixed point. 
Lemma 11 (see [10]). Let $\phi_{p}$ be a p-Laplacian operator. Then

(i) if $1<p \leq 2, \kappa_{1} \kappa_{2}>0$, and $\left|\kappa_{1}\right|,\left|\kappa_{2}\right| \geq m>0$, then

$$
\left|\phi_{p}\left(\kappa_{1}\right)-\phi_{p}\left(\kappa_{2}\right)\right| \leq(p-1) m^{p-2}\left|\kappa_{1}-\kappa_{2}\right| ;
$$

(ii) if $p>2$ and $\left|\kappa_{1}\right|,\left|\kappa_{2}\right| \leq M$, then

$$
\left|\phi_{p}\left(\kappa_{1}\right)-\phi_{p}\left(\kappa_{2}\right)\right| \leq(p-1) M^{p-2}\left|\kappa_{1}-\kappa_{2}\right| .
$$

Definition 12. Let $\mathbb{T}: \mathscr{L} \rightarrow \mathscr{L}$. Then the operator equation given by

$$
\mathbb{T} \mu(x)=\mu(x), \quad x \in[0,1]
$$

is called Hyers-Ulam stable if, for any $\xi>0$, the inequality given as

$$
\| \mu-\mathbb{}-\mathbb{} \mu \leq \xi, \quad x \in[0,1]
$$

has a unique fixed point say $\mu^{*}$ with constant $D>0$ such that $\left\|\mu-\mu^{*}\right\| \leq D \xi$, for all $x \in[0,1]$ holds.

In view of Definition 12, we give the following definition.

Definition 13. The system of Hammerstein type integral equations

$$
\begin{aligned}
\mu(x)= & \int_{0}^{1} \Omega_{\alpha_{1}}(x, \tau) \phi_{q}\left(I^{\beta_{1}} \mathfrak{F}_{1}(\tau, v(\tau))\right) d \tau \\
& +\frac{\eta_{1} x}{\Gamma\left(\gamma_{1}\right)} \int_{0}^{P}(P-\tau)^{\gamma_{1}-1} \psi_{1}(\mu(\tau)) d \tau, \\
v(x)= & \int_{0}^{1} \Omega_{\alpha_{2}}(x, \tau) \phi_{q}\left(I^{\beta_{2}} \mathfrak{F}_{2}(\tau, \mu(\tau))\right) d \tau \\
& +\frac{\eta_{2} x}{\Gamma\left(\gamma_{2}\right)} \int_{0}^{P}(P-\tau)^{\gamma_{2}-1} \psi_{2}(v(\tau)) d \tau
\end{aligned}
$$

is called Hyers-Ulam stable such that, for $D_{i}>0(i=1,2,3,4)$ and for all $\xi_{1}, \xi_{2}>0$ and for every solution $\left(\mu^{*}, v^{*}\right)$ to the system

$$
\begin{gathered}
\mid \mu^{*}(x)-\int_{0}^{1} \Omega_{\alpha_{1}}(x, \tau) \phi_{q}\left(I^{\beta_{1}} \mathfrak{F}_{1}(\tau, v(\tau))\right) d \tau \\
+\frac{\eta_{1} x}{\Gamma\left(\gamma_{1}\right)} \int_{0}^{P}(P-\tau)^{\gamma_{1}-1} \psi_{1}(\mu(\tau)) d \tau \mid \leq \xi_{1}, \\
\mid v^{*}(x)-\int_{0}^{1} \Omega_{\alpha_{2}}(x, \tau) \phi_{q}\left(I^{\beta_{2}} \mathfrak{F}_{2}(\tau, \mu(\tau))\right) d \tau \\
+\frac{\eta_{2} x}{\Gamma\left(\gamma_{2}\right)} \int_{0}^{P}(P-\tau)^{\gamma_{2}-1} \psi_{2}(v(\tau)) d \tau \mid \leq \xi_{2},
\end{gathered}
$$

there exists a unique solution $\left(\lambda_{1}, \lambda_{2}\right)$ of (21) satisfying the following system of inequalities:

$$
\begin{gathered}
\left\|\lambda_{1}-\mu^{*}\right\| \leq D_{1} \xi_{1}+D_{2} \xi_{2}, \quad x \in[0,1], \\
\left\|\lambda_{2}-v^{*}\right\| \leq D_{3} \xi_{1}+D_{4} \xi_{2}, \quad x \in[0,1] .
\end{gathered}
$$

\section{Some Data Dependence Assumptions}

To proceed further, let the following hypothesis hold:

$\left(\mathbb{A}_{1}\right)$ The nonlocal functions $\psi_{1}$ and $\psi_{2}$ where $\omega, \mu, \nu, v \in \mathbb{R}$ satisfy the following:

$$
\begin{gathered}
\left|\psi_{1}(\omega)-\psi_{1}(\mu)\right| \leq \mathbb{K}_{\psi_{1}}|\omega-\mu|, \\
\left|\psi_{2}(\nu)-\psi_{2}(v)\right| \leq \mathbb{K}_{\psi_{2}}|\nu-v|,
\end{gathered}
$$

where $\mathbb{K}_{\psi_{1}}, \mathbb{K}_{\psi_{2}} \in[0,1)$.

$\left(\mathbb{A}_{2}\right)$ To satisfy the following growth conditions by the constants $\mathbb{C}_{\psi_{1}}, \mathbb{C}_{\psi_{2}}, \mathbb{M}_{\psi_{1}}, \mathbb{M}_{\psi_{2}}>0$ and $q_{1} \in[0,1)$ for $\mu, v \in \mathbb{R}$ with the nonlocal functions $\psi_{1}$ and $\psi_{2}$ we have

$$
\begin{gathered}
\left|\psi_{1}(\mu)\right| \leq \mathbb{C}_{\psi_{1}}|\mu|^{q_{1}}+\mathbb{M}_{\psi_{1}}, \\
\left|\psi_{2}(v)\right| \leq \mathbb{C}_{\psi_{2}}|v|^{q_{1}}+\mathbb{M}_{\psi_{2}} .
\end{gathered}
$$

$\left(\mathbb{A}_{3}\right)$ The functions $\mathfrak{F}_{1}$ and $\mathfrak{F}_{2}$ satisfy the following growth conditions under the constants $a, b, \mathbb{M}_{1}^{*}, \mathbb{M}_{2}^{*}, p_{1} \in$ $(0,1]$ :

$$
\begin{gathered}
\left|\mathfrak{F}_{1}(x, \mu)\right| \leq a|\mu|^{p_{1}}+\mathbb{M}_{\mathfrak{F}_{1}}^{*}, \\
\left|\mathfrak{F}_{2}(x, v)\right| \leq b|v|^{p_{1}}+\mathbb{M}_{\mathfrak{F}_{2}}^{*} .
\end{gathered}
$$

$\left(\mathbb{A}_{4}\right)$ There exist real valued constants $\mathbb{L}_{\mathfrak{F}_{1}}$ and $\mathbb{L}_{\mathfrak{F}_{2}}$, and for all $\mu, v, \omega_{1}, \omega_{2} \in \mathbb{R}$,

$$
\begin{aligned}
& \left|\mathfrak{F}_{1}(x, v)-\psi_{1}\left(x, \omega_{1}\right)\right| \leq \mathbb{L}_{\mathfrak{F}_{1}}\left|v-\omega_{1}\right|, \\
& \left|\mathfrak{F}_{2}(x, \mu)-\psi_{2}\left(x, \omega_{2}\right)\right| \leq \mathbb{L}_{\mathfrak{\mho}_{1}}\left|\mu-\omega_{2}\right| .
\end{aligned}
$$

\section{Main Results}

Theorem 14. Let $\mathfrak{F}_{1}(x) \in C[0,1]$ be integrable function for FDEs and with integral boundary conditions; then the solution of

$$
\begin{aligned}
& \mathrm{D}^{\beta_{1}} \phi_{p}\left(\mathrm{D}^{\alpha_{1}} \mu(x)\right)+\mathfrak{F}_{1}(x)=0, \\
& \quad 0<\beta_{1} \leq 1,2<\alpha_{1} \leq 3, x \in[0,1], \\
& \left.\mathrm{D}^{\alpha_{1}} \mu(x)\right|_{x=0}=\left.\mu(x)\right|_{x=0}=\left.\mu^{\prime \prime}(x)\right|_{x=0}=0, \\
& \left.\mu^{\prime}(x)\right|_{x=1}=\eta_{1} I_{T}^{\gamma_{1}} \psi_{1}(\mu) \\
& \quad=\frac{\eta_{1}}{\Gamma\left(\gamma_{1}\right)} \int_{0}^{P}(P-\tau)^{\gamma_{1}-1} \psi_{1}(\mu(\tau)) d \tau
\end{aligned}
$$

is provided by

$$
\begin{aligned}
\mu(x)= & \int_{0}^{1} \Omega_{\alpha_{1}}(x, \tau) \phi_{q}\left(I^{\beta_{1}} \mathfrak{F}_{1}(\tau)\right) d \tau \\
& +\frac{\eta_{1} x}{\Gamma\left(\gamma_{1}\right)} \int_{0 q}^{P}(P-\tau)^{\gamma_{1}-1} \psi_{1}(\mu(\tau)) d \tau,
\end{aligned}
$$


where $\Omega_{\alpha_{1}}(x, \tau)$ is Green's function, given by

$$
\begin{aligned}
& \Omega_{\alpha_{1}}(x, \tau) \\
& = \begin{cases}\frac{x(1-\tau)^{\alpha_{1}-2}}{\Gamma\left(\alpha_{1}-1\right)}-\frac{(x-\tau)^{\alpha_{1}-1}}{\Gamma\left(\alpha_{1}\right)}, & 0 \leq \tau \leq x \leq 1, \\
\frac{x(1-\tau)^{\alpha_{1}-2}}{\Gamma\left(\alpha_{1}-1\right)}, & 0 \leq x \leq \tau \leq 1 .\end{cases}
\end{aligned}
$$

Proof. Applying the operator $I^{\beta_{1}}$ on (28) and using Lemma 3, we get from problem (28) the following equivalent integral form as

$$
\phi_{p}\left(D^{\alpha_{1}} \mu(x)\right)=-I^{\beta_{1}} \mathfrak{F}_{1}(x)+A_{0}
$$

By using conditions $\left.\mathrm{D}^{\alpha_{1}} \mu(x)\right|_{x=0}=0$, we get $A_{0}=0$. From (31), we have

$$
\mathrm{D}^{\alpha_{1}} \mu(x)=-\phi_{q}\left(I^{\beta_{1}} \mathfrak{F}_{1}(x)\right)
$$

Applying the operator $I^{\alpha_{1}}$ on (32) and using Lemma 3 again, we get from problem (32) the following equivalent integral form given by

$$
\mu(x)=-\mathscr{I}^{\alpha_{1}}\left(\phi_{q} I^{\beta_{1}} \mathfrak{\mho}_{1}(x)\right)+A_{1}+A_{2} x+A_{3} x^{2}
$$

By using the condition $\left.\mu(x)\right|_{x=0}=\left.\mu^{\prime \prime}(x)\right|_{x=0}=0$ in (33), we obtain $A_{1}=A_{3}=0$. Also in view of condition $\left.\mu^{\prime}(x)\right|_{x=1}=$ $\eta_{1} I_{T}^{\gamma} \psi_{1}(\mu)$ in (33), we get

$$
\begin{aligned}
A_{2}= & \frac{1}{\Gamma\left(\alpha_{1}-1\right)} \int_{0}^{1}(1-\tau)^{\alpha_{1}-2} \phi_{q}\left(I^{\beta_{1}} \mathfrak{\mho}_{1}(x)\right) d \tau \\
& +\frac{\eta_{1}}{\Gamma \gamma_{1}} \int_{0}^{P}(P-\tau)^{\gamma_{1}-1} \psi_{1}(\mu(\tau)) d \tau .
\end{aligned}
$$

By substituting the values of $A_{1}, A_{2}$, and $A_{3}$ in (33), we get the following integral equation:

$$
\begin{aligned}
& \mu(x)=\left[\frac{1}{\Gamma\left(\alpha_{1}-1\right)} \int_{0}^{1} x(1-\tau)^{\alpha_{1}-2}\right. \\
& \left.-\frac{1}{\Gamma\left(\alpha_{1}\right)} \int_{0}^{x}(x-\tau)^{\alpha_{1}-1}\right] \phi_{q}\left(I^{\beta_{1}} \mathfrak{\mho}_{1}(\tau)\right) d \tau \\
& +\frac{\eta_{1} x}{\Gamma\left(\gamma_{1}\right)} \int_{0}^{P}(P-\tau)^{\gamma_{1}-1} \psi_{1}(\mu(\tau)) d \tau \\
& =\int_{0}^{1} \Omega_{\alpha_{1}}(x, \tau) \phi_{q}\left(I^{\beta_{1}} \mathfrak{\mho}_{1}(\tau)\right) d \tau+\frac{\eta_{1} x}{\Gamma\left(\gamma_{1}\right)} \\
& \cdot \int_{0}^{P}(P-\tau)^{\gamma_{1}-1} \psi_{1}(\mu(\tau)) d \tau .
\end{aligned}
$$

According to Theorem 14, the equivalent system of Hammerstein type integral equations corresponding to coupled system (6) is given by

$$
\begin{aligned}
\mu(x)= & \int_{0}^{1} \Omega_{\alpha_{1}}(x, \tau) \phi_{q}\left(I^{\beta_{1}} \mathfrak{F}_{1}(\tau, v(\tau))\right) d \tau \\
& +\frac{\eta_{1} x}{\Gamma\left(\gamma_{1}\right)} \int_{0}^{P}(P-\tau)^{\gamma_{1}-1} \psi_{1}(\mu(\tau)) d \tau, \\
v(x)= & \int_{0}^{1} \Omega_{\alpha_{2}}(x, \tau) \phi_{q}\left(I^{\beta_{2}} \mathfrak{F}_{2}(\tau, \mu(\tau))\right) d \tau \\
& +\frac{\eta_{2} x}{\Gamma\left(\gamma_{2}\right)} \int_{0}^{P}(P-\tau)^{\gamma_{2}-1} \psi_{2}(v(\tau)) d \tau,
\end{aligned}
$$

where $\Omega_{\alpha_{2}}(x, \tau)$ is defined as

$$
\begin{aligned}
& \Omega_{\alpha_{2}}(x, \tau) \\
& = \begin{cases}\frac{x(1-\tau)^{\alpha_{2}-2}}{\Gamma\left(\alpha_{2}-1\right)}-\frac{(x-\tau)^{\alpha_{2}-1}}{\Gamma\left(\alpha_{2}\right)}, & 0 \leq \tau \leq x \leq 1, \\
\frac{x(1-\tau)^{\alpha_{2}-2}}{\Gamma\left(\alpha_{2}-1\right)}, & 0 \leq x \leq \tau \leq 1 .\end{cases}
\end{aligned}
$$

From $\Omega_{\alpha_{1}}(x, \tau)$ and $\Omega_{\alpha_{2}}(x, \tau)$ clearly,

$$
\begin{aligned}
& \max _{x \in J}\left|\Omega_{\alpha_{1}}(x, \tau)\right|=\frac{(1-\tau)^{\alpha_{1}-2}}{\Gamma\left(\alpha_{1}-1\right)}, \\
& \max _{t \in J}\left|\Omega_{\alpha_{2}}(x, \tau)\right|=\frac{(1-\tau)^{\alpha_{2}-2}}{\Gamma\left(\alpha_{2}-1\right)} .
\end{aligned}
$$

Further, we define the operators $\varphi_{1}: \mathscr{L}_{1} \rightarrow \mathscr{L}_{1}$ and $\varphi_{2}:$ $\mathscr{L}_{2} \rightarrow \mathscr{L}_{2}$ by

$$
\varphi_{1} \mu(x)=\frac{\eta_{1} x}{\Gamma\left(\gamma_{1}\right)} \int_{0}^{P}(P-\tau)^{\gamma_{1}-1} \psi_{1}(\mu(\tau)) d \tau,
$$$$
x \in[0,1],
$$$$
\varphi_{2} v(x)=\frac{\eta_{2} x}{\Gamma\left(\gamma_{2}\right)} \int_{0}^{P}(P-\tau)^{\gamma_{2}-1} \psi_{2}(v(\tau)) d \tau
$$

$$
x \in[0,1],
$$

and $\Upsilon_{i}: \mathscr{L} \rightarrow \mathscr{L}$ for $(i=1,2)$ by

$$
\begin{gathered}
\Upsilon_{1} v(x)=\int_{0}^{1} \Omega_{\alpha_{1}}(x, \tau) \phi_{q}\left(I^{\beta_{1}} \mathfrak{F}_{1}(\tau, v(\tau))\right) d \tau, \\
x \in[0,1] \\
\Upsilon_{2} \mu(x)=\int_{0}^{1} \Omega_{\alpha_{2}}(x, \tau) \phi_{q}\left(I^{\beta_{2}} \mathfrak{F}_{2}(\tau, \mu(\tau))\right) d \tau, \\
x \in[0,1] .
\end{gathered}
$$

Hence we have $\varphi(\mu, v)=\left(\varphi_{1}, \varphi_{2}\right)(\mu, v), \Upsilon(\mu, v)=\left(\Upsilon_{1}, \Upsilon_{2}\right)$ $(\mu, v)$, and $\mathbb{T}(\mu, v)=\varphi(\mu, v)+\Upsilon(\mu, v)$. Therefore the operator 
equation of Hammerstein type integral equations (36) is given by

$$
(\mu, v)=\mathbb{T}(\mu, v)=\varphi(\mu, v)+\Upsilon(\mu, v) .
$$

Thus the solution of Hammerstein type equation (36) is the fixed points of operator equation (41).

Theorem 15. In view of hypotheses $\left(\mathbb{A}_{1}\right)$ and $\left(\mathbb{A}_{4}\right)$, the operator $\varphi$ is $\mathrm{j}$-Lipschitz and satisfies the growth condition given by

$$
\|\varphi(\mu, v)\| \leq \mathbb{C}_{\varphi}\|(\mu, v)\|^{q_{1}}+\mathbb{M}_{\varphi}, \quad \forall(\mu, v) \in \mathscr{L} .
$$

Proof. From condition $\left(\mathbb{A}_{1}\right)$ and using $x \leq 1$, we get

$$
\begin{aligned}
& \left|\varphi_{1}(\mu)(x)-\varphi_{1}(\bar{\mu})(x)\right| \\
& \quad=\left|\frac{\eta_{1}}{\Gamma\left(\gamma_{1}\right)} \int_{0}^{P}(P-\tau)^{\gamma_{1}-1}\left[\psi_{1}(\mu)-\psi_{1}(\bar{\mu})\right] d \tau\right| \\
& \quad \leq \frac{\eta_{1}}{\Gamma\left(\gamma_{1}\right)} \int_{0}^{P}(P-\tau)^{\gamma_{1}-1}\left|\psi_{1}(\mu)-\psi_{1}(\bar{\mu})\right| d \tau,
\end{aligned}
$$

which implies that $\left|\varphi_{1}(\mu)(x)-\varphi_{2}(\bar{\mu})(x)\right| \leq \overline{\mathbb{K}}_{\psi_{1}}\|\mu-\bar{\mu}\|$, where $\overline{\mathbb{K}}_{\psi_{1}}=\mathbb{K}_{\psi_{1}} P^{\gamma_{1}} / \Gamma\left(\gamma_{1}+1\right) \in[0,1)$. To obtain the growth condition we have

$$
\begin{aligned}
\left|\varphi_{1}(\mu)(x)\right| & =\left|\frac{\eta_{1}}{\Gamma\left(\gamma_{1}\right)} \int_{0}^{P}(P-\tau)^{\gamma_{1}-1} \psi_{1}(\mu(\tau)) d \tau\right| \\
& \leq \frac{\eta_{1}}{\Gamma\left(\gamma_{1}\right)} \int_{0}^{P}(P-\tau)^{\gamma_{1}-1}\left|\psi_{1}(\mu(\tau))\right| d \tau .
\end{aligned}
$$

Upon simplification, we get from (44)

$$
\left\|\varphi_{1} \mu\right\| \leq \frac{\eta_{1} P^{\gamma_{1}}}{\Gamma\left(\gamma_{1}+1\right)}\left[\mathbb{C}_{\psi_{1}}\|\mu\|^{q_{1}}+\mathbb{M}_{\psi_{1}}\right] .
$$

Similarly, we get

$$
\left\|\varphi_{2} v\right\| \leq \frac{\eta_{2} P^{\gamma_{2}}}{\Gamma\left(\gamma_{2}+1\right)}\left[\mathbb{C}_{\psi_{2}}\|v\|^{q_{1}}+\mathbb{M}_{\psi_{2}}\right] .
$$

Now

$$
\begin{aligned}
& \|\varphi(\mu, v)\| \\
& \leq \frac{\eta_{1} P^{\gamma_{1}}}{\Gamma\left(\gamma_{1}+1\right)}\left[\mathbb{C}_{\psi_{1}}\|\mu\|^{q_{1}}+\mathbb{M}_{\psi_{1}}\right] \\
& \quad+\frac{\eta_{2} P^{\gamma_{2}}}{\Gamma\left(\gamma_{2}+1\right)}\left[\mathbb{C}_{\psi_{2}}\|v\|^{q_{1}}+\mathbb{M}_{\psi_{2}}\right] \\
& \leq\left(\frac{\eta_{1} P^{\gamma_{1}}}{\Gamma\left(\gamma_{1}+1\right)} \mathbb{C}_{\psi_{1}}\|\mu\|^{q_{1}}+\frac{\eta_{2} P^{\gamma_{2}}}{\Gamma\left(\gamma_{2}+1\right)} \mathbb{C}_{\psi_{2}}\|v\|^{q_{1}}\right) \\
& \quad+\left(\frac{\eta_{1} P^{\gamma_{1}} \mathbb{M}_{\psi_{1}}}{\Gamma\left(\gamma_{1}+1\right)}+\frac{\eta_{2} P^{\gamma_{2}} \mathbb{M}_{\psi_{2}}}{\Gamma\left(\gamma_{2}+1\right)}\right) \\
& \leq \mathbb{C}_{\varphi}\left[\|\mu\|^{q_{1}}+\|v\|^{q_{1}}\right]+\mathbb{M}_{\varphi}=\mathbb{C}_{\varphi}\|(\mu, v)\|^{q_{1}}+\mathbb{M}_{\varphi},
\end{aligned}
$$

where

$$
\begin{aligned}
& \max \left\{\frac{\eta_{1} P^{\gamma_{1}}}{\Gamma\left(\gamma_{1}+1\right)} \mathbb{C}_{\psi_{1}}, \frac{\eta_{2} P^{\gamma_{2}}}{\Gamma\left(\gamma_{2}+1\right)} \mathbb{C}_{\psi_{2}}\right\}=\mathbb{C}_{\varphi}, \\
& \mathbb{M}_{\varphi}=\frac{\eta_{1} P^{\gamma_{1}} \mathbb{M}_{\psi_{1}}}{\Gamma\left(\gamma_{1}+1\right)}+\frac{\eta_{2} P^{\gamma_{2}} \mathbb{M}_{\psi_{2}}}{\Gamma\left(\gamma_{2}+1\right)} .
\end{aligned}
$$

Theorem 16. In view of hypothesis $\left(\mathbb{A}_{3}\right)$, the operator $\Upsilon$ is continuous and satisfies the growth condition given by

$$
\|\Upsilon(\mu, v)\|^{p_{1}} \leq F\|(\mu, v)\|^{p_{1}}+\Xi,
$$

where $F=\wp(a+b), \wp=\max \left\{\left((q-1) m_{1}^{q-2} / \Gamma\left(\beta_{1}+1\right)\right)\left(1 / \Gamma\left(\alpha_{1}+\right.\right.\right.$ $\left.1)),\left((q-1) m_{2}^{q-2} / \Gamma\left(\beta_{2}+1\right)\right)\left(1 / \Gamma\left(\alpha_{2}+1\right)\right)\right\}$, and $\Xi=\wp\left(\mathbb{M}_{1}^{*}+\mathbb{M}_{2}^{*}\right)$ for each $(\mu, v) \in \mathscr{L}$.

Proof. Consider bounded set $\mathbb{B}_{r}=\{(\mu, v) \in \mathscr{L}:\|(\mu, v)\| \leq r\}$ with sequence $\left(\mu_{n}, v_{n}\right)$ converging to $(\mu, v)$ in $\mathbb{B}_{r}$. We have to show that $\left\|\Upsilon\left(\mu_{n}, v_{n}\right)-\Upsilon(\mu, v)\right\| \rightarrow 0$ as $n \rightarrow \infty$. Therefore, we have to consider

$$
\begin{aligned}
& \left|\Upsilon_{1}\left(v_{n}(x)\right)-\Upsilon(v(x))\right| \leq \mid \frac{x}{\Gamma\left(\alpha_{1}-1\right)} \int_{0}^{1}(1-\tau)^{\alpha_{1}-2} \\
& \cdot\left[\phi_{q}\left(I^{\beta} \mathfrak{F}_{1}\left(\tau, v_{n}(\tau)\right)\right)\right. \\
& \left.\quad-\phi_{q}\left(I^{\beta} \mathfrak{F}_{1}(\tau, v(\tau))\right)\right] d \tau|+| \frac{1}{\Gamma\left(\alpha_{1}\right)} \int_{0}^{x}(x \\
& -\tau)^{\alpha_{1}-1}\left[\phi_{q}\left(I^{\beta} \mathfrak{F}_{1}\left(\tau, v_{n}(\tau)\right)\right)\right. \\
& \left.-\phi_{q}\left(I^{\beta} \mathfrak{\mho}_{1}(\tau, v(\tau))\right)\right] d \tau \mid \leq(q-1) \\
& \cdot m_{1}^{q-2}\left[\frac{1}{\Gamma\left(\alpha_{1}-1\right)} \int_{0}^{1}(1-\tau)^{\alpha_{1}-2}-\frac{1}{\Gamma\left(\alpha_{1}\right)} \int_{0}^{x}(x\right. \\
& \left.-\tau)^{\alpha_{1}-1}\right] \times\left|I^{\beta} \mathfrak{\mho}_{1}\left(\tau, v_{n}(\tau)\right)-I^{\beta} \mathfrak{\Im}_{1}(\tau, v(\tau))\right| d \tau .
\end{aligned}
$$

Due to continuity of $\mathfrak{F}_{1}$, one has $\mathfrak{F}_{1}\left(\tau, v_{n}(\tau)\right) \rightarrow \mathfrak{F}_{1}(\tau, v(\tau))$ as $n \rightarrow \infty$. Thus in view of Lebesgue dominated convergent theorem, we have $\left|I^{\beta} \mathfrak{F}_{1}\left(x, v_{n}(x)\right)-I^{\beta} \mathfrak{F}_{1}(x, v(x))\right| \rightarrow 0$ as $n \rightarrow \infty$. Thus $\left|\Upsilon_{1}\left(v_{n}(x)\right)-\Upsilon(v(x))\right| \rightarrow 0$, as $n \rightarrow \infty$. Hence $\Upsilon_{1}$ is continuous. Now for growth condition (49), we have

$$
\begin{aligned}
& \left|\Upsilon_{1} v(x)\right|=\left|\int_{0}^{1} \Omega_{\alpha_{1}}(x, \tau) \phi_{q}\left(I^{\beta_{1}} \mathfrak{F}_{1}(\tau, v(\tau))\right) d \tau\right| \\
& \quad \leq(q-1) m_{1}^{q-2}\left|\int_{0}^{1} \Omega_{\alpha_{1}}(x, \tau)\left(I^{\beta_{1}} \mathfrak{F}_{1}(\tau, v(\tau))\right) d \tau\right|
\end{aligned}
$$

$\left\|\Upsilon_{1} v\right\|^{p_{1}}$

$$
\leq \frac{(q-1) m_{1}^{q-2}}{\Gamma\left(\beta_{1}+1\right)}\left[\frac{1}{\Gamma\left(\alpha_{1}+1\right)}\right]\left(a\|v\|^{p_{1}}+\mathbb{M}_{1}^{*}\right),
$$




$$
\begin{aligned}
& \left|\Upsilon_{2} \mu(x)\right|=\left|\int_{0}^{1} \Omega_{\alpha_{2}}(x, \tau) \phi_{q}\left(I^{\beta_{2}} \widetilde{\mho}_{2}(\tau, \mu(\tau))\right) d s\right| \\
& \quad \leq(q-1) m_{2}^{q-2}\left|\int_{0}^{1} \Omega_{\alpha_{2}}(x, \tau)\left(I^{\beta_{2}} \mathfrak{\mho}_{1}(\tau, v(\tau))\right) d s\right|, \\
& \left\|\Upsilon_{2} \mu\right\|^{p_{1}} \\
& \quad \leq \frac{(q-1) m_{2}^{q-2}}{\Gamma\left(\beta_{2}+1\right)}\left[\frac{1}{\Gamma\left(\alpha_{2}+1\right)}\right]\left(b\|\mu\|^{p_{1}}+\mathbb{M}_{2}^{*}\right) .
\end{aligned}
$$

From, (51), we obtain the following result

$$
\begin{aligned}
\|\Upsilon(\mu, v)\|^{p_{1}}= & \left\|\Upsilon_{1}(v)\right\|^{p_{1}}+\left\|\Upsilon_{2}(\mu)\right\|^{p_{1}} \\
\leq & \wp\left(a\|v\|^{p_{1}}+\mathbb{M}_{1}^{*}\right)+\wp\left(b\|\mu\|^{p_{1}}+\mathbb{M}_{2}^{*}\right) \\
\leq & \wp(a+b)\left(\|v\|^{p_{1}}+\|\mu\|^{p_{1}}\right) \\
& +\wp\left(\mathbb{M}_{1}^{*}+\mathbb{M}_{2}^{*}\right)=F\|(\mu, v)\|^{p_{1}}+\Xi .
\end{aligned}
$$

Theorem 17. The operator $\Upsilon: \mathscr{L} \rightarrow \mathscr{L}$ is compact and $\mathrm{j}$ Lipschitz with constant zero.

Proof. Consider a sequence $\left\{\mathfrak{F}_{n}\right\}$ in bounded set $D$ such that $D$ is subset of $\mathbb{B}_{r}$; in view of (49), we see

$$
\left\|\Upsilon\left(\mu_{n}, v_{n}\right)\right\|^{p_{1}} \leq F\left\|\left(\mu_{n}, v_{n}\right)\right\|^{p_{1}}+\Xi
$$

$$
\text { for every }(\mu, v) \in \mathscr{L} \text {. }
$$

Thus $\Upsilon$ is bounded for $\left(\mu_{n}, v_{n}\right) \in D$. Let us, for any $x_{1}, x_{2} \in$ $[0,1]$, consider

$$
\begin{aligned}
& \left|Y v_{n}\left(x_{1}\right)-\Upsilon v_{n}\left(x_{2}\right)\right| \\
& \quad=\mid \int_{0}^{1} \Omega_{\alpha_{1}}\left(x_{1}, \tau\right) \phi_{q}\left(I^{\beta_{1}} \mathfrak{\mho}_{1}\left(\tau, v_{n}(\tau)\right)\right) d \tau \\
& \quad-\int_{0}^{1} \Omega_{\alpha_{1}}\left(x_{2}, \tau\right) \phi_{q}\left(I^{\beta_{1}} \mathfrak{\mho}_{1}\left(\tau, v_{n}(\tau)\right)\right) d \tau \mid \leq(q \\
& \quad-1) m_{1}^{q-2}\left|\int_{0}^{1} \Omega_{1}\left(x_{1}, \tau\right)-\int_{0}^{1} \Omega_{1}\left(x_{2}, \tau\right)\right| \\
& \quad \cdot\left|\left(I^{\beta_{1}} \mathfrak{\mho}_{1}\left(\tau, v_{n}(\tau)\right)\right)\right| d \tau \\
& \quad \leq \frac{(q-1) m_{1}^{q-2}}{\Gamma\left(\beta_{1}+1\right)}\left[\frac{\left(x_{1}-x_{2}\right)}{\Gamma\left(\alpha_{1}-1\right)} \int_{0}^{1}(1-\tau)^{\alpha_{1}-2} d \tau\right. \\
& \quad+\frac{1}{\Gamma\left(\alpha_{1}\right)}\left(\int_{0}^{x_{1}}\left(x_{1}-\tau\right)^{\alpha_{1}-1} d \tau\right. \\
& \left.\left.-\int_{0}^{x_{2}}\left(x_{2}-\tau\right)^{\alpha_{1}-1} d \tau\right)\right]\left(a\left\|v_{n}\right\|^{p_{1}}+\mathbb{M}_{1}^{*}\right) .
\end{aligned}
$$

By simplification, we get the following result:

$$
\begin{aligned}
& \left|\Upsilon_{1} v_{n}\left(x_{1}\right)-\Upsilon_{1} v_{n}\left(x_{2}\right)\right| \\
& \quad \leq \frac{(q-1) m_{1}^{q-2}}{\Gamma\left(\beta_{1}+1\right)}\left[\frac{\left|x_{1}-x_{2}\right|}{\Gamma\left(\alpha_{1}\right)}+\frac{\left|x_{1}^{\alpha_{1}}-x_{2}^{\alpha_{1}}\right|}{\Gamma\left(\alpha_{1}+1\right)}\right] \\
& \cdot\left(a\left\|\mu_{n}\right\|^{p_{1}}+\mathbb{M}_{1}^{*}\right) .
\end{aligned}
$$

And also in same fashion, one has

$$
\begin{aligned}
& \left|\Upsilon_{2} \mu_{n}\left(x_{1}\right)-\Upsilon_{2} \mu_{n}\left(x_{2}\right)\right| \\
& \quad \leq \frac{(q-1) m_{2}^{q-2}}{\Gamma\left(\beta_{2}+1\right)}\left[\frac{\left|x_{1}-x_{2}\right|}{\Gamma\left(\alpha_{2}\right)}+\frac{\left|x_{1}^{\alpha_{2}}-x_{2}^{\alpha_{2}}\right|}{\Gamma\left(\alpha_{2}+1\right)}\right] \\
& \cdot\left(b\|v\|^{p_{2}}+\mathbb{M}_{2}^{*}\right) .
\end{aligned}
$$

Therefore if $x_{1} \rightarrow x_{2}$, then the right hand side of both sides of (55) and (56) tends to zero. Thus $\Upsilon_{1}, \Upsilon_{2}$ are equicontinuous, and therefore $\Upsilon=\left(\Upsilon_{1}, \Upsilon_{2}\right)$ is equicontinuous on $D$. Hence, thanks to the Arzelá-Ascoli theorem, $\Upsilon(D)$ is compact. Also, by proposition $\Upsilon$ is $j$-Lipschitz with constant zero.

Remark 18. The results of Theorems 15, 16, and 17 are also hold for using $q_{1}=p_{1}=1$.

Theorem 19. In view of assumptions $\left(\mathbb{A}_{1}\right)-\left(\mathbb{A}_{4}\right)$ such that $\mathbb{C}_{\varphi}+$ $F \leq 1$, then the coupled system (6) has at least one solution $(\mu, v) \in \mathscr{L}$ together with condition that the set of the solutions $\mathscr{B}$ is bounded in $\mathscr{L}$.

Proof. $\varphi$ and $\Upsilon$ are continuous and $\jmath$-Lipschitz with constant $\mathbb{C}_{\varphi}$ and 0 , respectively. Thanks to Theorem $10, \mathbb{T}$ is strict $j$ contraction, and then consider a set

$$
\mathscr{B}=\{(\mu, v) \in \mathscr{L}: \lambda \in[0,1] \mid(\mu, v)=\lambda \mathbb{T}(\mu, v)\} .
$$

To prove that $\mathscr{B}$ is bounded, let

$$
\begin{aligned}
\|(\mu, v)\| & =\|\lambda \mathbb{T}(\mu, v)\| \leq\|\lambda \varphi(\mu, v)\|+\|\lambda \Upsilon(\mu, v)\| \\
& \leq \mathbb{C}_{\varphi}\|(\mu, v)\|+\mathbb{M}_{\varphi}+F\|(\mu, v)\|+\Xi \\
& =\left(\mathbb{C}_{\varphi}+F\right)\|(\mu, v)\|+\mathbb{M}_{\varphi}+\Xi .
\end{aligned}
$$

Therefore, the set of solutions $\mathscr{B}$ is bounded.

Theorem 20. Assume that hypotheses $\left(\mathbb{A}_{1}\right)-\left(\mathbb{A}_{4}\right)$ hold. Then system (6) has a unique solution if and only if $\Theta<1$, where

$$
\begin{aligned}
\Theta= & \frac{\eta_{1} \mathbb{K}_{\psi_{1}} P^{\gamma_{1}}}{\Gamma\left(\gamma_{1}+1\right)}+\frac{\eta_{2} \mathbb{K}_{\psi_{2}} T^{\gamma_{2}}}{\Gamma\left(\gamma_{2}+1\right)}+\frac{(q-1) m_{1}^{q-2} \mathbb{L}_{\widetilde{\mho}_{1}}}{\Gamma\left(\beta_{1}+1\right) \Gamma\left(\alpha_{1}\right)} \\
& +\frac{(q-1) m_{2}^{q-2} \mathbb{L}_{\widetilde{\mho}_{2}} .}{\Gamma\left(\beta_{2}+1\right) \Gamma \alpha_{2}} .
\end{aligned}
$$

Proof. Let $(\mu, v)$ and $(\bar{\mu}, \bar{v}) \in \mathscr{L}$ be two solutions; then

$$
\begin{aligned}
|\mathbb{T}(\mu, v)-\mathbb{T}(\bar{\mu}, \bar{v})| \leq & |\varphi(\mu, v)-\varphi(\bar{\mu}, \bar{v})| \\
& +|\Upsilon(\mu, v)-\Upsilon(\bar{\mu}, \bar{v})| ;
\end{aligned}
$$


therefore by simplification (60), we get the following result:

$$
\begin{aligned}
\| & \mathbb{T}(\mu, v)-\mathbb{T}(\bar{\mu}, \bar{v}) \| \leq\left(\frac{\eta_{1} \mathbb{K}_{\psi_{1}} P^{\gamma_{1}}}{\Gamma\left(\gamma_{1}+1\right)}+\frac{\eta_{2} \mathbb{K}_{\psi_{2}} T^{\gamma_{2}}}{\Gamma\left(\gamma_{2}+1\right)}\right. \\
+ & \left.\frac{(q-1) m_{1}^{q-2} \mathbb{L}_{\mathfrak{\mho}_{1}}}{\Gamma\left(\beta_{1}+1\right) \Gamma \alpha_{1}}+\frac{(q-1) m_{2}^{q-2} \mathbb{L}_{\mathfrak{\mho}_{2}}}{\Gamma\left(\beta_{2}+1\right) \Gamma \alpha_{2}}\right) \|(\mu, v) \\
& -(\bar{\mu}, \bar{v})\|\leq \Theta\|(\mu, v)-(\bar{\mu}, \bar{v}) \| .
\end{aligned}
$$

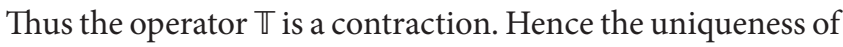
solution to system (6) follows due to the Banach fixed point theorem.

\section{Hyers-Ulam Stability of Coupled System}

In the present section, we derive the Hyers-Ulam type stability for the solution of the considered problem.

Theorem 21. By assumptions $\left(\mathbb{A}_{1}\right)-\left(\mathbb{A}_{4}\right)$, system (6) is HyersUlam stable.

Proof. By Theorem 20 and Definition 13, let $\left(\lambda_{1}, \lambda_{2}\right)$ be the exact solution, and let $\left(\mu^{*}, v^{*}\right)$ be any other solution of system (36). Then from equation of (36) we have

$$
\begin{gathered}
\left|\lambda_{1}(x)-\mu^{*}(x)\right| \leq \mid \int_{0}^{1} \Omega_{\alpha_{1}}(x, \tau) \phi_{q} \\
\cdot\left[I^{\beta_{1}}\left(\mathfrak{F}_{1}(\tau, y(\tau))-\mathfrak{F}_{1}\left(\tau, v^{*}(\tau)\right)\right)\right] d \tau+\frac{\eta_{1} x}{\Gamma\left(\gamma_{1}\right)} \\
\cdot \int_{0}^{P}(P-\tau)^{\gamma_{1}-1}\left(\psi_{1}(x)-\psi_{1}\left(\mu^{*}\right)\right) d \tau \mid \\
\text { implies that }\left\|\lambda_{1}-\mu^{*}\right\| \leq \frac{(q-1) m_{1}^{q-2} \mathbb{L}_{\mathfrak{F}_{1}}}{\Gamma\left(\beta_{1}+1\right) \Gamma\left(\alpha_{1}\right)}\left\|x-v^{*}\right\| \\
+\frac{\eta_{1} P^{\gamma_{1}} K_{\psi_{1}}}{\Gamma\left(\gamma_{1}+1\right)}\left\|y-\mu^{*}\right\| \leq D_{1} \xi_{1}+D_{2} \xi_{2},
\end{gathered}
$$

where $D_{1}=(q-1) m_{1}^{q-2} \mathbb{L}_{\widetilde{F}_{1}} / \Gamma\left(\beta_{1}+1\right) \Gamma\left(\alpha_{1}\right)$ and $D_{2}=$ $\eta_{1} P^{\gamma_{1}} \mathbb{K}_{\psi_{1}} / \Gamma\left(\gamma_{1}+1\right)$. By the same process we also get that

$$
\left\|\lambda_{2}-v^{*}\right\| \leq D_{3} \xi_{1}+D_{4} \xi_{2}
$$

where $D_{3}=(q-1) m_{1}^{q-2} \mathbb{L}_{\mathfrak{\mho}_{2}} / \Gamma\left(\beta_{2}+1\right) \Gamma\left(\alpha_{2}\right)$ and $D_{4}=$ $\eta_{2} P^{\gamma_{2}} \mathbb{K}_{\psi_{2}} / \Gamma\left(\gamma_{2}+1\right)$.

Hence in view of (62) and (64), the system of integral equation (36) is Hyers-Ulam stable, and consequently, the solution of system (6) is Hyers-Ulam stable.

\section{Illustrative Examples}

Example 1. Consider the following coupled system of FDEs with $p$-Laplacian operator and fractional order differential and integral boundary conditions of the type

$$
\begin{aligned}
& \mathrm{D}^{1 / 2} \phi_{p}\left(\mathrm{D}^{5 / 2} \mu(x)\right)+\left(\frac{x}{99}+19 \sin |\nu(x)|\right)=0 \\
& \mathrm{D}^{1 / 2} \phi_{p}\left(\mathrm{D}^{5 / 2} v(x)\right)+\left(\frac{x}{100}+3 \sin |\mu(x)|+75\right)=0 \\
& \left.\mathrm{D}^{5 / 2} \mu(x)\right|_{x=0}=0=\left.\mu(x)\right|_{x=0}=\left.\mu^{\prime \prime}(x)\right|_{x=0}, \\
& \left.\mu^{\prime}(x)\right|_{x=1}=\frac{1}{3 \Gamma(3 / 2)} \int_{0}^{1}(1-\tau)^{1 / 2} \cos (\mu) d \tau \\
& \left.\mathrm{D}^{5 / 2} v(x)\right|_{x=0}=0=\left.v(x)\right|_{x=0}=\left.v^{\prime \prime}(x)\right|_{x=0} \\
& \left.v^{\prime}(x)\right|_{x=1}=\frac{1}{3 \Gamma(3 / 2)} \int_{0}^{1}(1-\tau)^{1 / 2} \cos (v) d \tau
\end{aligned}
$$

where $x \in[0,1], P=1, p=3, \alpha_{i}=5 / 2, \beta_{i}=1 / 2, \eta_{i}=1 / 3$, $\gamma_{i}=3 / 2$, where $i=1,2, \mathbb{K}_{\psi_{1}}, \mathbb{K}_{\psi_{2}}=1 / 5, \mathbb{M}_{i}=2$, and $\mathbb{L}_{\mathfrak{F}_{i}}=$ $1 / 6$. By simple calculation we obtain the following:

$$
\begin{aligned}
& \frac{(1 / 3)(1 / 5)}{\Gamma(3 / 2+1)}+\frac{(1 / 3)(1 / 5)}{\Gamma(3 / 2+1)}+\frac{(3 / 2-1) 2^{-1 / 2}(1 / 6)}{\Gamma(1 / 2+1) \Gamma(5 / 2)} \\
& +\frac{(3 / 2-1) 2^{-1 / 2}(1 / 6)}{\Gamma(1 / 2+1) \Gamma(5 / 2)}<1 .
\end{aligned}
$$

Therefor from Theorem 20, we concluded that (65) has unique solution. Similarly, the conditions of Theorem 21 can be verified easily. Thus the solution of system (65) is HyersUlam stable.

\section{Conclusion}

We have investigated sufficient conditions for existence and uniqueness of solutions to a coupled system of nonlinear FDEs with fractional integral boundary conditions with nonlinear $p$-Laplacian operator by using topological degree method. Further, we have established some adequate conditions for the Hyers-Ulam stability to the proposed problem.

\section{Conflicts of Interest}

The authors declare that no conflicts of interest exist regarding this manuscript.

\section{Authors' Contributions}

All authors equally contributed to this paper and approved the final version.

\section{Acknowledgments}

This work has been supported by the National Natural Science Foundation of China (11571378). 


\section{References}

[1] I. Podlubny, Fractional Differential Equations, vol. 198 of Mathematics in Science and Engineering, Academic Press, San Diego, Calif, USA, 1999.

[2] R. Hilfer, Applications of Fractional Calculus in Physics, World Scientific, Singapore, 2000.

[3] A. A. Kilbas, H. M. Srivastava, and J. J. Trujillo, Theory and Applications of Fractional Differential Equations, New York, NY, USA, Elsevier, 2006.

[4] K. S. Miller and B. Ross, An Introduction to the Fractional Calculus and Fractional Differential Equations, Wiley, New York, NY, USA, 1993.

[5] K. B. Oldham, "Fractional differential equations in electrochemistry," Adv. Eng. Soft, vol. 41, p. 12, 2010.

[6] L. Diening, P. Lindqvist, and B. Kawohl, "Mini-Workshop: The p-laplacian operator and applications," Oberwol-fach Reports, vol. 10, no. 1, pp. 433-482, 2013.

[7] H. Lu, Z. Han, and S. Sun, "Multiplicity of positive solutions for Sturm-Liouville boundary value problems of fractional differential equations with $p$-LAPlacian," Boundary Value Problems, 2014:26, 17 pages, 2014.

[8] L. Hu and S. Zhang, "On existence results for nonlinear fractional differential equations involving the $p$-Laplacian at resonance," Mediterr. J. Mth, vol. 10, pp. 015-014, 2015.

[9] E. Zhi, X. Liu, and F. Li, "Nonlocal boundary value problem for fractional differential equations with $p$-Laplacian," Mathematical Methods in the Applied Sciences, vol. 37, no. 17, pp. 2651-2662, 2014.

[10] T. Shen, W. Liu, and X. Shen, "Existence and uniqueness of solutions for several BVPs of fractional differential equations with $p$ Laplacian operator," Mediterranean Journal of Mathematics, vol. 13, no. 6, pp. 4623-4637, 2016.

[11] L. Zhang, W. Zhang, X. Liu, and M. Jia, "Existence of positive solutions for integral boundary value problems of fractional differential equations with $p$-Laplacian," Advances in Difference Equations, Paper No. 36, 19 pages, 2017.

[12] R. A. Khan, A. Khan, A. Samad, and H. Khan, "On existence of solutions for fractional differential equation with p-Laplacian operator," Journal of Fractional Calculus and Applications, vol. 5, no. 2, pp. 28-37, 2014.

[13] E. Cetin and F. S. Topal, "Existence of solutions for fractional four point boundary value problems with p-Laplacian operator," Journal of Computational Analysis and Applications, vol. 19, no. 5, pp. 892-903, 2015.

[14] X. Liu, M. Jia, and W. Ge, “The method of lower and upper solutions for mixed fractional four-point boundary value problem with $p$-Laplacian operator," Applied Mathematics Letters. An International Journal of Rapid Publication, vol. 65, pp. 56-62, 2017.

[15] I. M. Stamova, "Mittag-Leffler stability of impulsive differential equations of fractional order," Quarterly of Applied Mathematics, vol. 73, no. 3, pp. 525-535, 2015.

[16] F. Isaia, "On a nonlinear integral equation without compactness," Acta Mathematica Universitatis Comenianae. New Series, vol. 75, no. 2, pp. 233-240, 2006.

[17] J. Wang, Y. Zhou, and W. Wei, "Study in fractional differential equations by means of topological degree methods," Numerical Functional Analysis and Optimization. An International Journal, vol. 33, no. 2, pp. 216-238, 2012.
[18] K. Shah and R. A. Khan, "Existence and uniqueness results to a coupled system of fractional order boundary value problems by topological degree theory," Numerical Functional Analysis and Optimization. An International Journal, vol. 37, no. 7, pp. 887899, 2016.

[19] K. Shah, A. Ali, and R. A. Khan, "Degree theory and existence of positive solutions to coupled systems of multi-point boundary value problems," Boundary Value Problems, Paper No. 43, 12 pages, 2016.

[20] L. Gao, D. Wang, and G. Wang, "Further results on exponential stability for impulsive switched nonlinear time-delay systems with delayed impulse effects," Applied Mathematics and Computation, vol. 268, pp. 186-200, 2015.

[21] J. C. Trigeassou, N. Maamri, J. Sabatier, and A. Oustaloup, "A Lyapunov approach to the stability of fractional differential equations," Signal Processing, vol. 91, no. 3, pp. 437-445, 2011.

[22] S. M. Ulam, Problems in Modern Mathematics, Science Editions, Chapter 6, Wiley, New York, NY, USA, 1960.

[23] D. H. Hyers, "On the stability of the linear functional equation," Proceedings of the National Academy of Sciences of the United States of America, vol. 27, pp. 222-224, 1941.

[24] I. A. Rus, "Ulam stabilities of ordinary differential equations in a Banach space," Carpathian Journal of Mathematics, vol. 26, no. 1, pp. 103-107, 2010.

[25] S.-M. Jung, "Hyers-Ulam stability of a system of first order linear differential equations with constant coefficients," Journal of Mathematical Analysis and Applications, vol. 320, no. 2, pp. 549-561, 2006.

[26] S.-M. Jung and T. M. Rassias, "Generalized hyers-ulam stability of riccati differential equation," Mathematical Inequalities \& Applications, vol. 11, no. 4, pp. 777-782, 2008.

[27] C. Urs, "Coupled fixed point theorems and applications to periodic boundary value problems," Miskolc Mathematical Notes, vol. 14, no. 1, pp. 323-333, 2013.

[28] S. d. Abbas and M. Benchohra, "On the generalized UlamHyers-Rassias stability for Darboux problem for partial fractional implicit differential equations," Applied Mathematics ENotes, vol. 14, pp. 20-28, 2014.

[29] R. W. Ibrahim, "Stability for univalent solutions of complex fractional differential equations," Proceedings of the Pakistan Academy of Sciences, vol. 49, no. 3, pp. 227-232, 2012.

[30] A. Ali, B. Samet, K. Shah, and R. . Khan, "Existence and stability of solution to a toppled systems of differential equations of noninteger order," Boundary Value Problems, Paper No. 16, 13 pages, 2017.

[31] K. Deimling, Nonlinear Functional Analysis, Springer, Berlin , Germany, 1985. 


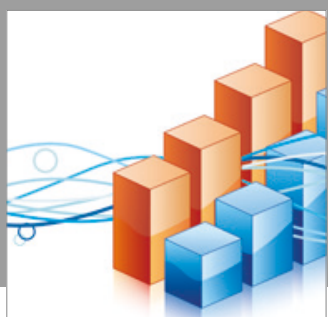

Advances in

Operations Research

vatersals

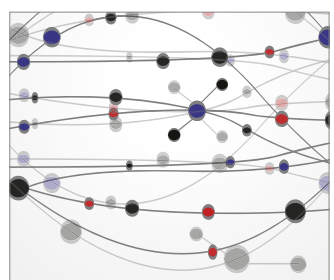

\section{The Scientific} World Journal
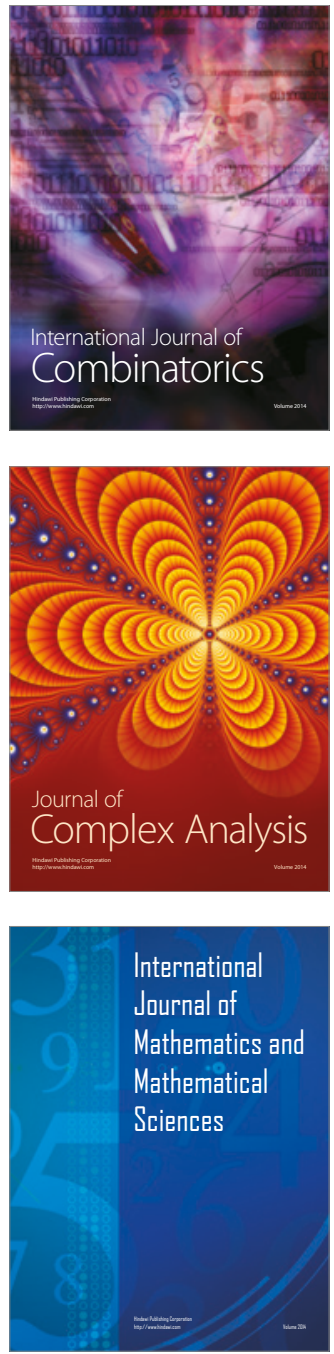
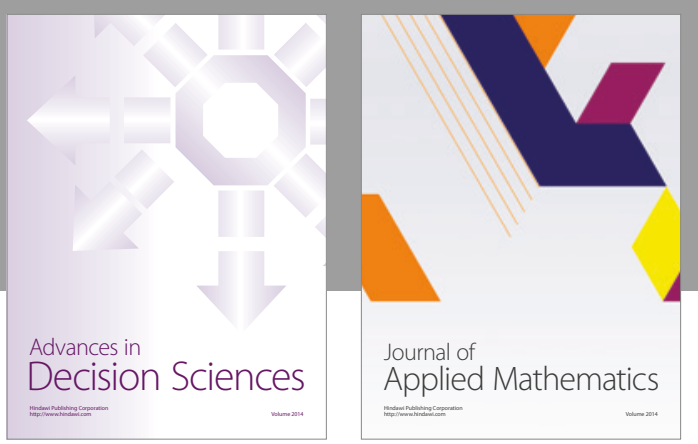

Algebra

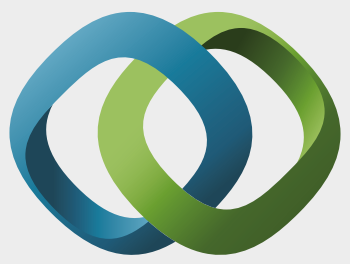

\section{Hindawi}

Submit your manuscripts at

https://www.hindawi.com
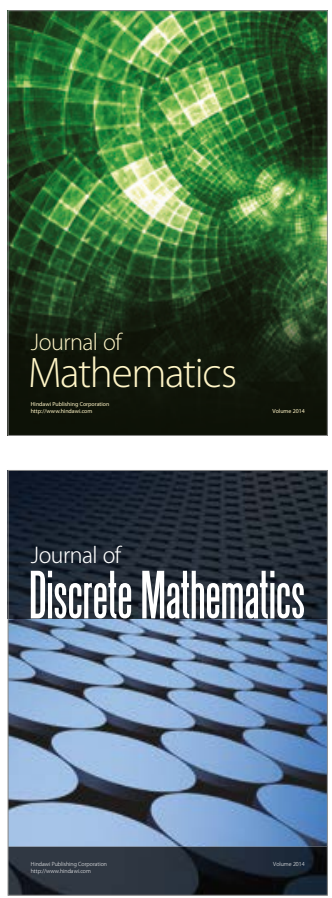

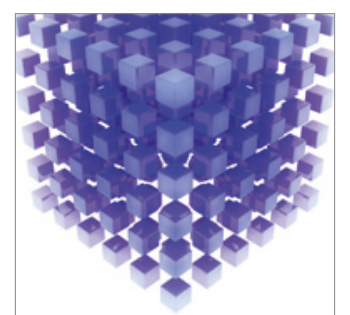

Mathematical Problems in Engineering
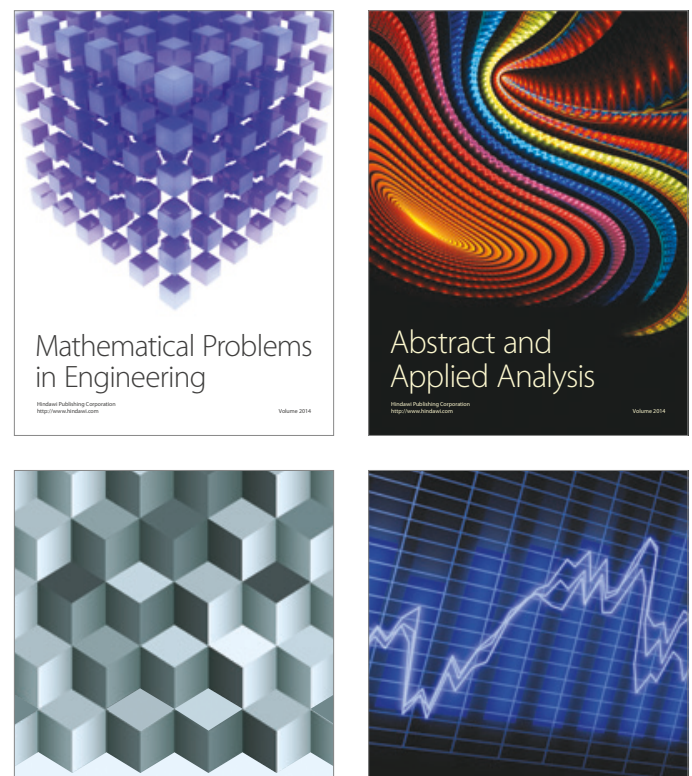

Journal of

Function Spaces

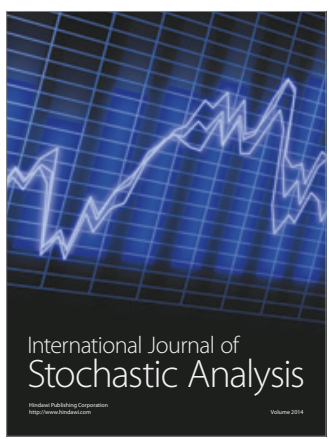

Probability and Statistics
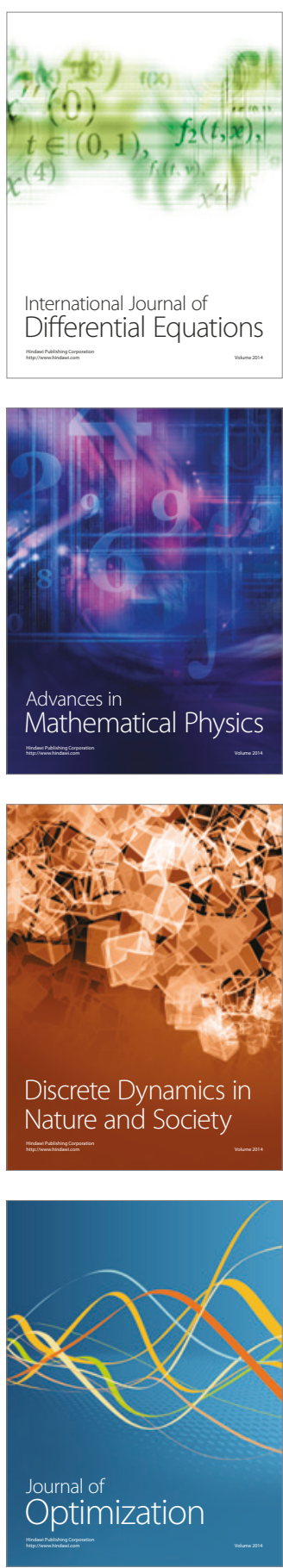